\title{
Contrast-enhanced MRI of aortal atherosclerosis: syndrome types and prediction of dissection
}

\author{
Alexandra S Maximova*, Vadim E Babokin, Irina L Bukhovets, Yevgeniya E Bobrikova, Yuliya V Rogovskaya, \\ Pavel I Lukyanenok, Wladimir Y Ussov \\ From 18th Annual SCMR Scientific Sessions \\ Nice, France. 4-7 February 2015
}

\section{Background}

Although magnetic resonance angiography is well established and deliver highly sensitive diagnosis of aneurismatic disease of aorta in patients with extensive atherosclerosis, the routine imaging of aortal wall with contrast-enhanced (CE) MRI itself is not well developed and not in routine clinical use.

\section{Methods}

The patients population comprised 33 patients (pts, 31 men, 2 women, aged $57 \pm 9$ years) with extensive atherosclerosis and old transmural acute myocardial infarction (AMI) of the left ventricle. All pts underwent cardiac CE MRI before coronary artery bypass surgery. As a control group eight patients with tumor pathology of the chest without evidence of clinically significant atherosclerosis were employed (aged $53 \pm 8$ years). In addition to the $\mathrm{CE}$ MRI of the heart, the CE MRI of atherosclerotic lesions of the aorta was carried out. Before and in 15-20 minutes after the injection of paramagnetic contrast agent T1weighted spin-echo images (TR=450-600 ms, TE=15 ms) were acquired. Geometric diameter and wall thickness of the aorta at the level of uptaken of contrast-paramagnetic agent to the wall, and index of enhancement (IE) were also measured.

\section{Results}

In the control group no significant accumulation of contrast paramagnetic material in the aortic wall was observed, IE did not exceed 1.04(mean $1.01 \pm 0.06$ ), the diameter and the wall thickness at the accumulation of contrast are $2.1 \pm 0.24 \mathrm{~cm}$ and $0.34 \pm 0.05 \mathrm{~cm}$, respectively. In $25(76 \%)$ patients with extensive atherosclerosis and

\footnotetext{
Federal State Budgetary Scientific Institution "Research Institute for
} Cardiology", Tomsk, Russian Federation

\section{Conclusions}

MRI CE technique of atherosclerotic lesions of the aorta can be suggested as additional independent research technique in atherosclerosis prospective studies of both surgical and medical methods of anti-atherosclerotic therapy. At the moment a study of the risk of delamination, aneurysmal rupture of atherosclerotic lesions is in progress, aimed at developing additional criteria to their surgical management.

Published: 3 February 2015

doi:10.1186/1532-429X-17-S1-P256

Cite this article as: Maximova et al:: Contrast-enhanced MRI of aortal atherosclerosis: syndrome types and prediction of dissection. Journal of Cardiovascular Magnetic Resonance 2015 17(Suppl 1):P256. 


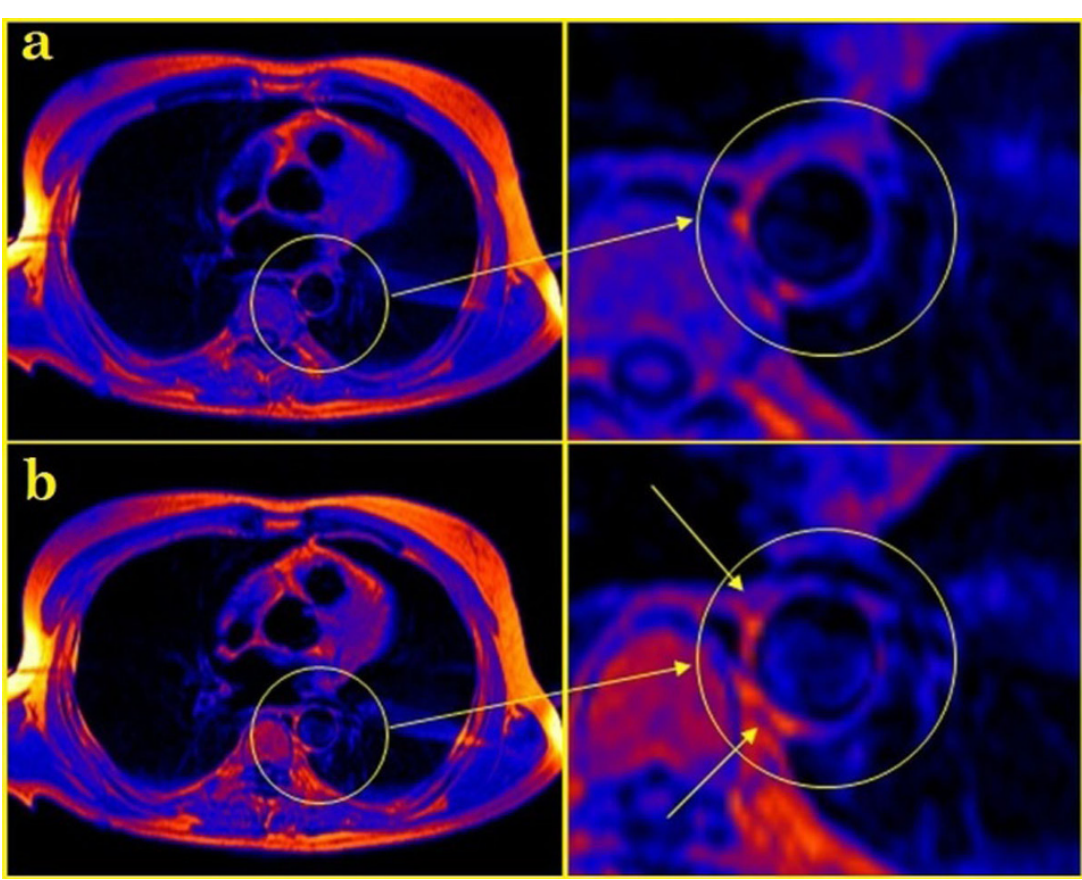

Figure $1 \mathrm{MRI}$ in patient with extensive atherosclerosis and myocardial infarction of the left ventricular: a - T1-weighted image before contrast injection, $b$ - T2-weighted image after contrasting - local accumulation of contrast-paramagnetic material in the aortic wall.

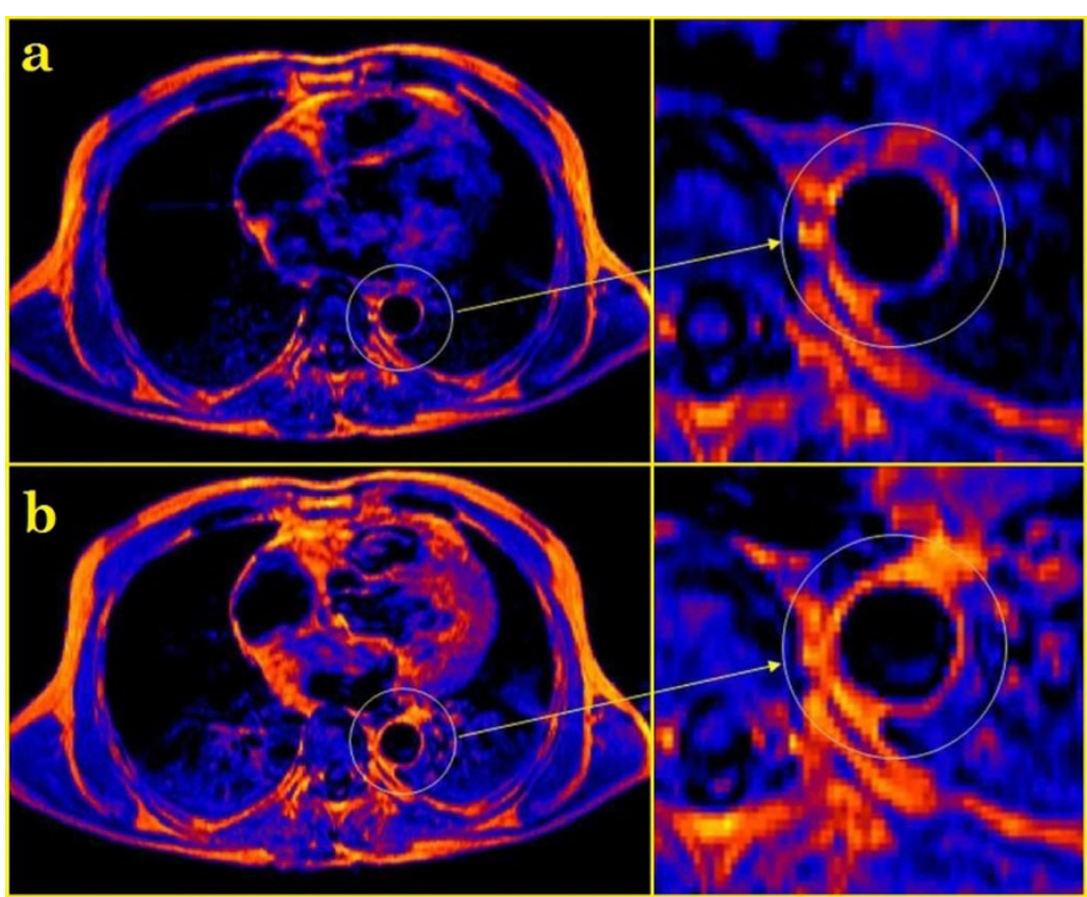

Figure $2 \mathrm{MRI}$ in patient with extensive atherosclerosis and myocardial infarction of the left ventricular - a - T1-weighted image before contrast injection, b-T2-weighted image after contrasting - diffuse accumulation syndrome of contrast paramagnetic material in the aortic wall. 